\title{
MITEQ DR-125G-A, 12-GHz FIBER-OPTIC DETECTOR EVALUATIONS FOR The Photonic Doppler Velocimetry Diagnostic
}

\author{
Araceli Rutkowski, Michael Rutkowski ${ }^{1}$ \\ National Security Technologies, LLC, Los Alamos Operations
}

The 12.5-GHz bandwidth MITEQ DR-125G-A detector is used often in the photonic Doppler velocimetry (PDV) diagnostic of Los Alamos National Laboratory (LANL). This paper presents detector characteristics as applied to optical heterodyning. We propose a test setup to simulate the beat frequency generated when incident and reflected light from a moving surface are mixed by optically combining a small-linewidth, tunable laser with a fixed, 1550-nm, thin-linewidth, high-power laser. The detector's transfer function, harmonic content, and signal-to-noise ratio (SNR) were to be measured and plotted for different optical power levels. Based on these results, appropriate light levels can be set to produce the highest dynamic range and signal level for the beat frequency. The goal is to provide insight into setting up the diagnostic for optimal detector performance using a specific optical input power.

\section{Introduction}

The PDV diagnostic has been deployed by LANL quite often in the last six months, with very good results. Setting up the PDV detector for optimal performance has been largely a trial-and-error process, in which each configuration has its share of unique issues. The MITEQ DR-125G-A detector consists of a PIN diode and a high-gain transimpedance amplifier. Its total responsivity is approximately $1900 \mathrm{~V} / \mathrm{W}$. The detector's 3-dB bandwidth ranges from $10 \mathrm{kHz}$ to $12.5 \mathrm{GHz}$, and maximum output can reach up to $3.5 \mathrm{~V}$. Each channel in the detector module system is often configured similarly to the block diagram shown in Figure 1. A high-power laser of 5 to $10 \mathrm{~W}$ is split to produce and input optical power from 0.5 to $2 \mathrm{~W}$ at $1550 \mathrm{~nm}$. An inline optical power meter monitors source power with only a 0.3-dB loss. The source light (also called "unshifted light") enters the optical circulator with a 0.77-dB loss to Port 2, then onto the 90/10 fused coupler. The through leg of the coupler has a 0.72-dB loss and is used as the output to the probe. The tap leg of the fused coupler has $11.5 \mathrm{~dB}$ of loss and generates unshifted light back into the detector. The unshifted light travels into the optical attenuator, with $1 \mathrm{~dB}$ of loss at a 0 -dB attenuation setting, to the 0.6-dB-loss, gold-capped optical reflector. From the reflector, the signal travels back to the attenuator to the tapped leg of the fused coupler (another $11.5 \mathrm{~dB}$ of loss) to Port 2 of the optical circulator. The unshifted light goes through to Port 3 ( $0.77 \mathrm{~dB}$ of loss), to a second power meter ( $0.41 \mathrm{~dB}$ of loss), to the MITEQ detector. The user can adjust the amount of unshifted light at the detector using the optical attenuator.

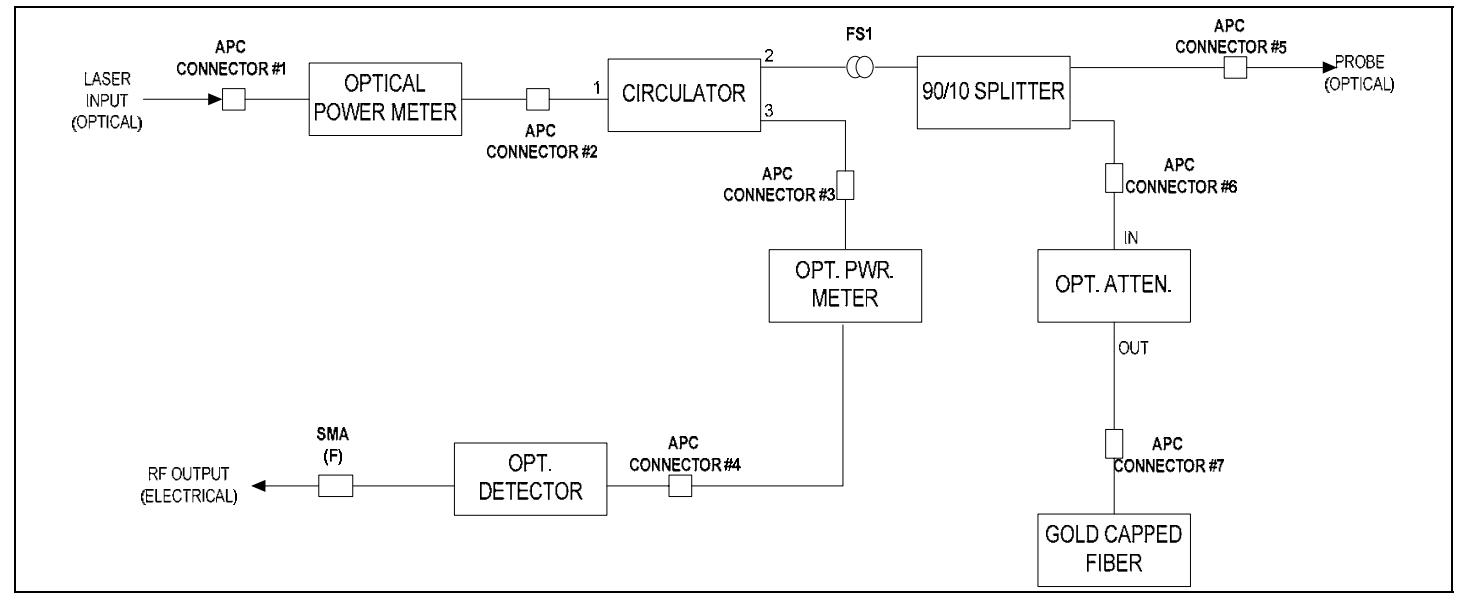

Figure 1. Typical PDV block diagram

${ }^{1}$ RutkowMS@nv.doe.gov, (505) 663-2074 
The incident light travels down some length of single-mode fiber (this may not always be the case but is desired). As the surface moves, a Doppler-shifted light is reflected back up the single-mode fiber to the through leg of the fused coupler ( $0.72 \mathrm{~dB}$ of loss). The Doppler-shifted light (also referred to as "shifted light") enters optical circulator Port 2 and exits Port 3 with a $0.77-\mathrm{dB}$ lower signal. It travels through the second inline power meter $(0.41 \mathrm{~dB}$ of loss) to the MITEQ detector.

The interference between the shifted and unshifted light produces a frequency at the detector output. Unshifted light at the detector can range from an estimated 0.5 to $20 \mathrm{~mW}$, while shifted light can vary from $10 \mathrm{nW}$ to $10 \mu \mathrm{W}$.

\section{Test Setup}

A test configuration was needed to generate both shifted and unshifted optical signals in the lab without having to use the Doppler-shifted light reflected from a moving surface. The basic requirements for the optical sources were: adjustable optical output power, good optical power and wavelength stability and a maximum wavelength difference between the optical sources of a few picometers. The test configuration shown in Figure 2 was set up and evaluated for use to characterize the MITEQ DR-125G-A detector. An IPG Photonics ELR-5-1550-SF laser generated the unshifted light. The randomly polarized laser has a $50-\mathrm{kHz}$ linewidth (FWHM). Its output power is $5 \mathrm{~W}$, scalable down to a few $\mathrm{mW}$. This laser is very similar to the laser source utilized in the PDV diagnostic. To generate the shifted light, we used the Agilent 81940A Compact Tunable Laser Source. This laser has a linewidth of $100 \mathrm{kHz}$ (FWHM) and a wavelength stability of $\pm 2.5 \mathrm{pm}$ over 24 hours. Its wavelength resolution is $1 \mathrm{pm}$ at $1550 \mathrm{~nm}$. Optical attenuators were connected to the output of each laser to adjust their output power levels. The IPG Photonics laser was connected to the JDS Uniphase VA4 optical attenuator, while the Agilent tunable laser was connected to the HP 8158B optical attenuator. The attenuators' outputs were fed into a JDS Uniphase 50/50 fused optical coupler. An EigenLight M320 series power meter was placed in line with the detector under test (DUT) to minimize fiber disruption during data recording. The EigenLight meter has an absolute accuracy of $\pm 0.2 \mathrm{~dB}$. The output of the DUT was connected to the Agilent E4440A high-performance spectrum analyzer, with a 26.5-GHz bandwidth and $140 \mathrm{~dB}$ of dynamic range. All fibers were single-mode SMF-28 9/125- $\mu \mathrm{m}$ Corning fiber. All fiber connections were FC/APC.

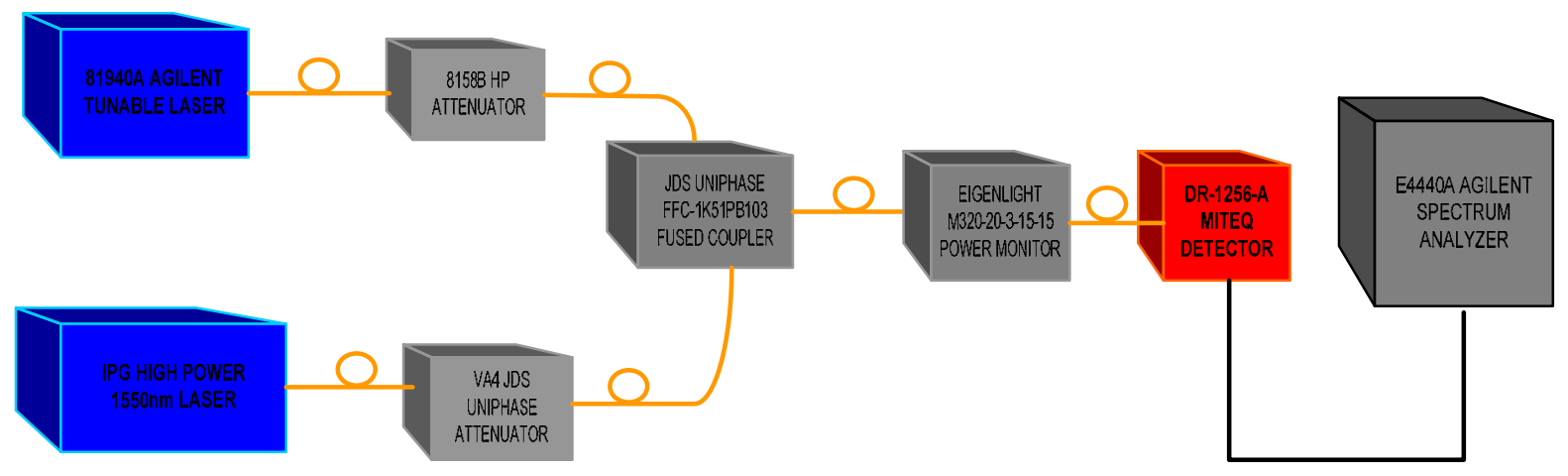

Figure 2. Test-setup block diagram

The IPG laser had a measured wavelength of $1549.944 \mathrm{~nm}$, while the tunable laser was set to $1549.974 \mathrm{~nm}$. These settings produced a beat frequency of $3.8 \pm 0.3 \mathrm{GHz}$. Spectrum analyzer settings were:

- Video bandwidth: $50 \mathrm{MHz}$

- Resolution bandwidth: $8 \mathrm{MHz}$

- Frequency span: $1 \mathrm{GHz}$

- Center frequency: $4 \mathrm{GHz}$. 
Because the 3.8-GHz signal amplitude varied by 2 to $3 \mathrm{~dB}$, we used the analyzer's Max Hold feature to obtain the reading. Due to polarization mismatch, the beat signal proved very sensitive in amplitude as the optical fiber cable was moved. It was stabilized by taping all fiber jumpers to the lab bench.

\section{Transfer Function}

To determine the optimum value of unshifted light into the detector, we measured the root-mean-square output voltage vs. unshifted optical power while holding the shifted light constant. Figure 3 shows the resulting transfer curves of the MITEQ detector, S/N\#1092539, when the shifted light was held constant at $100 \mathrm{nW}$, and the unshifted light was adjusted from 0 to $16 \mathrm{~mW}$. There was good correlation between the calculated and measured data, until the optical power reached $10 \mathrm{~mW}$. From 10 to $11.5 \mathrm{~mW}$, the amplitude leveled off to $78 \mathrm{mV}$, then declined to $40 \mathrm{mV}$, at an input power of $16 \mathrm{~mW}$. This data supports the conclusion that there is an optimum optical level at which one can achieve maximum output voltage.

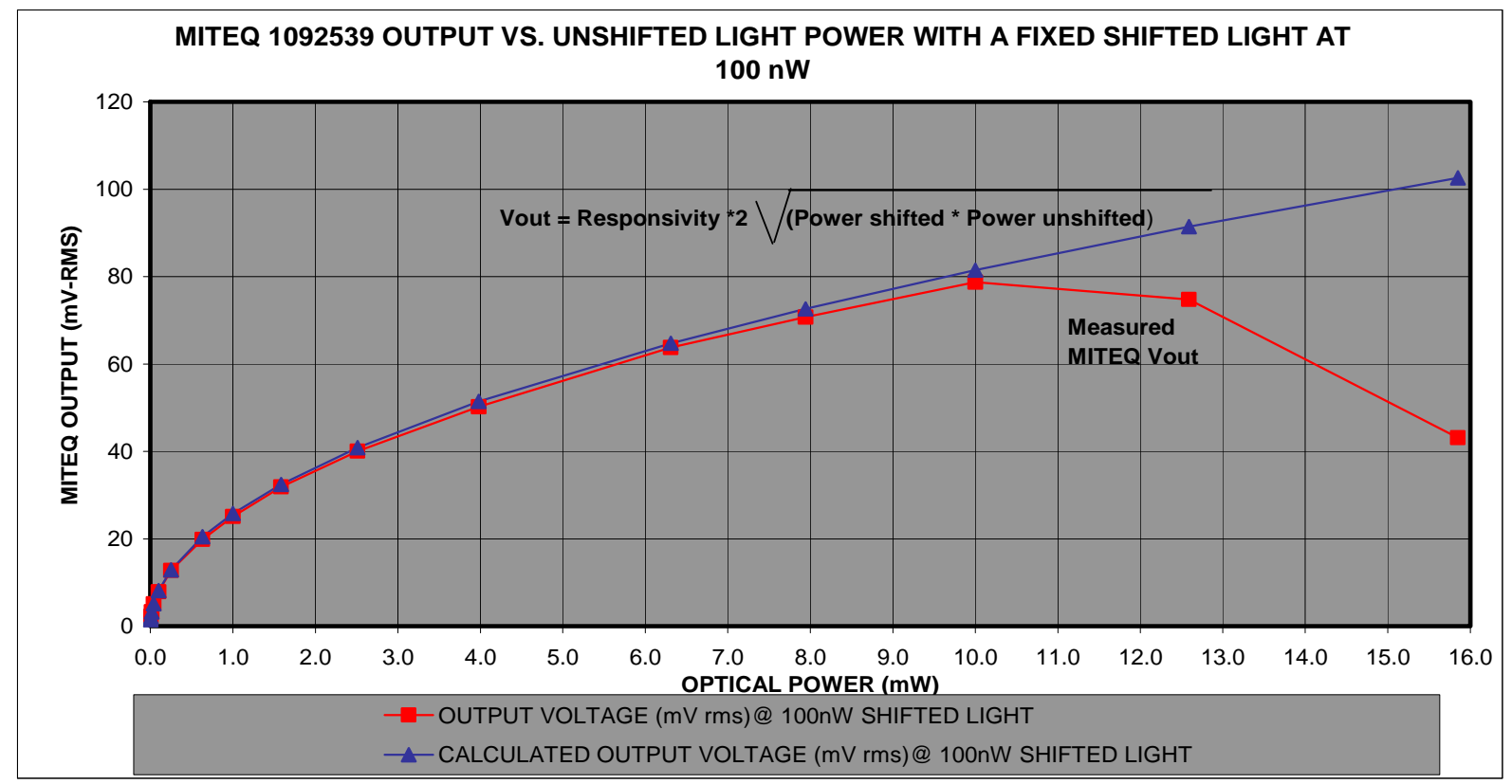

Figure 3. MITEQ transfer function calculated vs. measured

The graph in Figure 4 shows the same MITEQ detector with the output radio frequency (RF) level at four different shifted-light levels. In this case, the data demonstrates that the optimum input optical power level occurs at the same point independently of the shifted-light level. Figure 4 also demonstrates how quickly the output voltage declines with decreasing shifted-light levels. The graph in Figure 5 shows a closer view of the MITEQ transfer function at lower unshifted light power levels. 


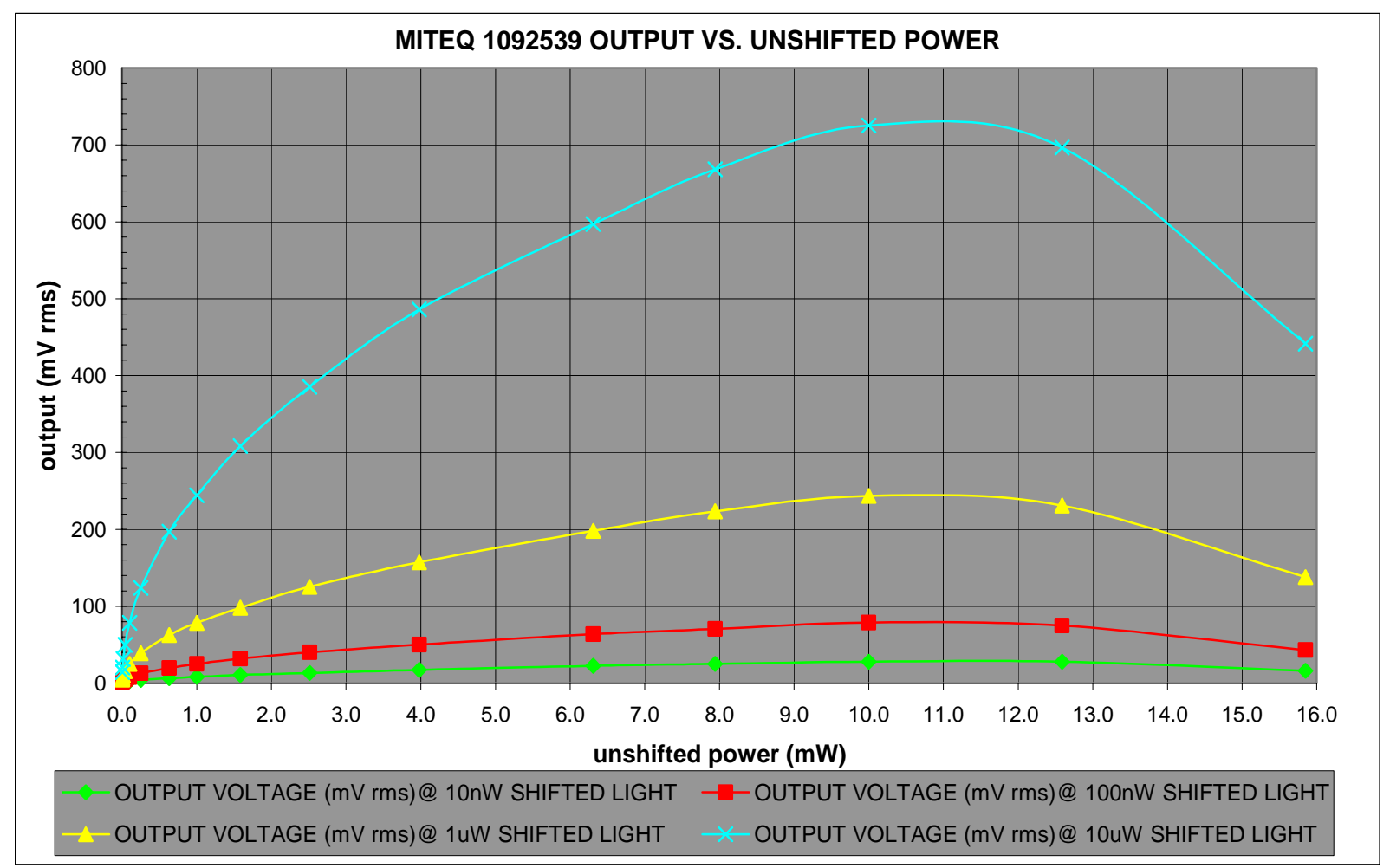

Figure 4. MITEQ measured transfer function for different shifted optical powers

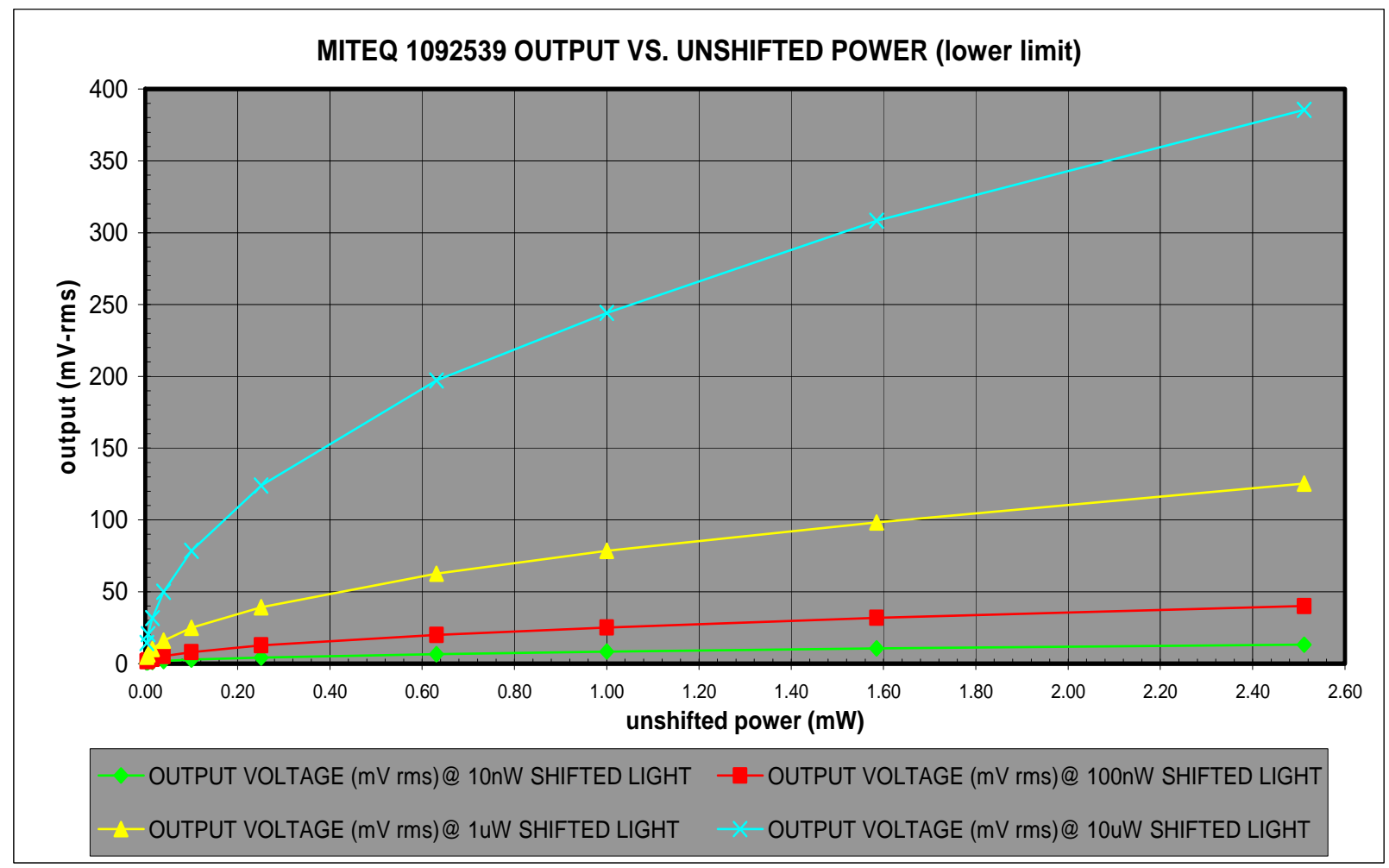

Figure 5. MITEQ transfer function at lower unshifted optical power 
The graph in Figure 6 plots a second MITEQ detector S/N\#1104469. This data show that, under the same test conditions, its output level at 10- $\mu \mathrm{W}$ shifted light (white trace) is $\sim 30 \%$ higher ( $250 \mathrm{mV}$ ) than the output signal of the MITEQ S/N\#1092539 (see Figure 5). Notice that the peaking of the output signal on S/N\#1104469 occurs at $3.5 \mathrm{~mW}$ lower than that of the S/N\#1092539. Based on this information, a total of four MITEQ detectors were evaluated under the same test conditions. Results appear in Figure 7. This graph shows that each MITEQ detector has its own "operational sweet spot” for optimum output voltage versus input optical power. This "spot” varies from 7 to $12 \mathrm{~mW}$ of input power and 80 to $120 \mathrm{mV}$ of output voltage, or possibly even more.

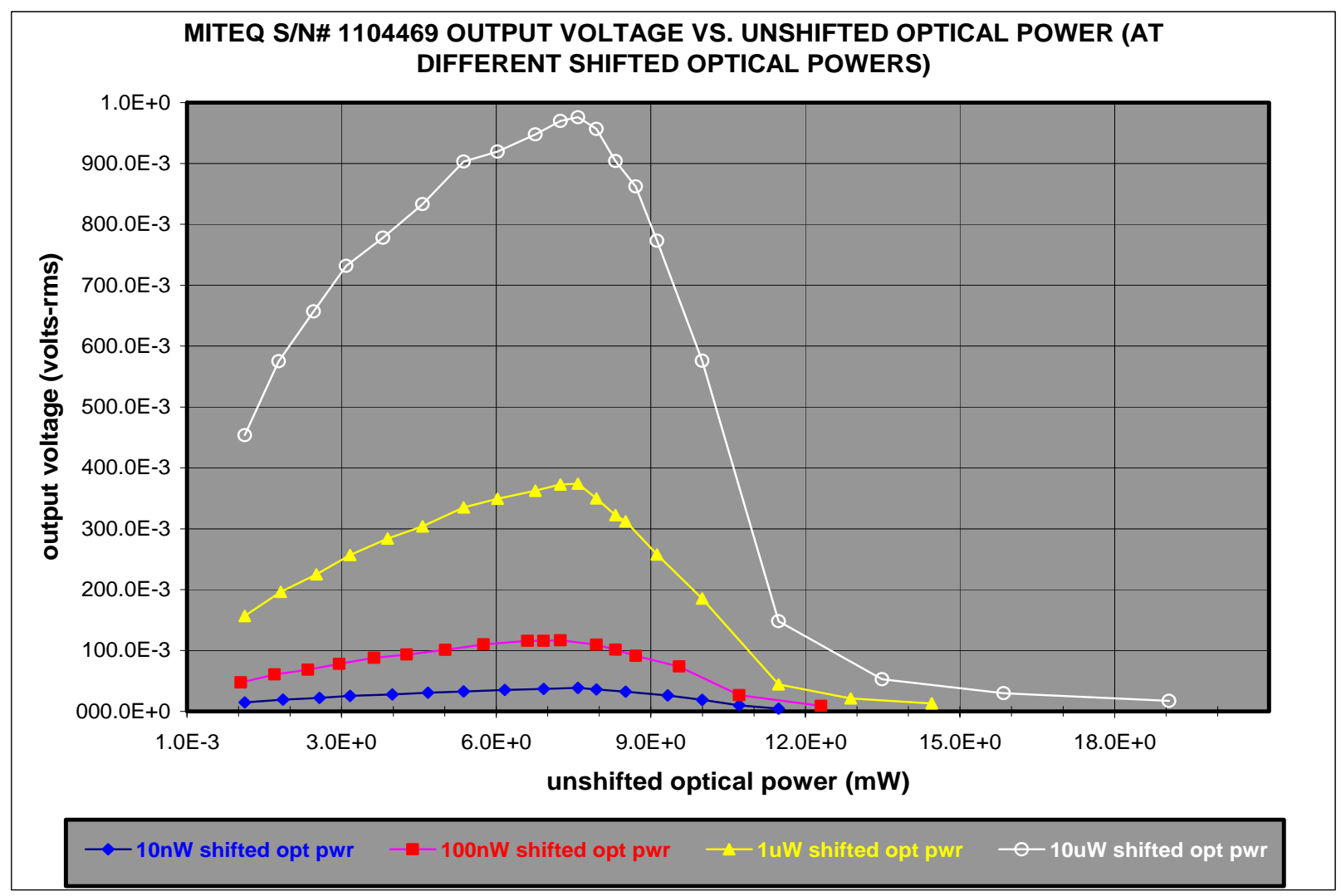

Figure 6. Second MITEQ detector transfer function at different shifted optical powers 


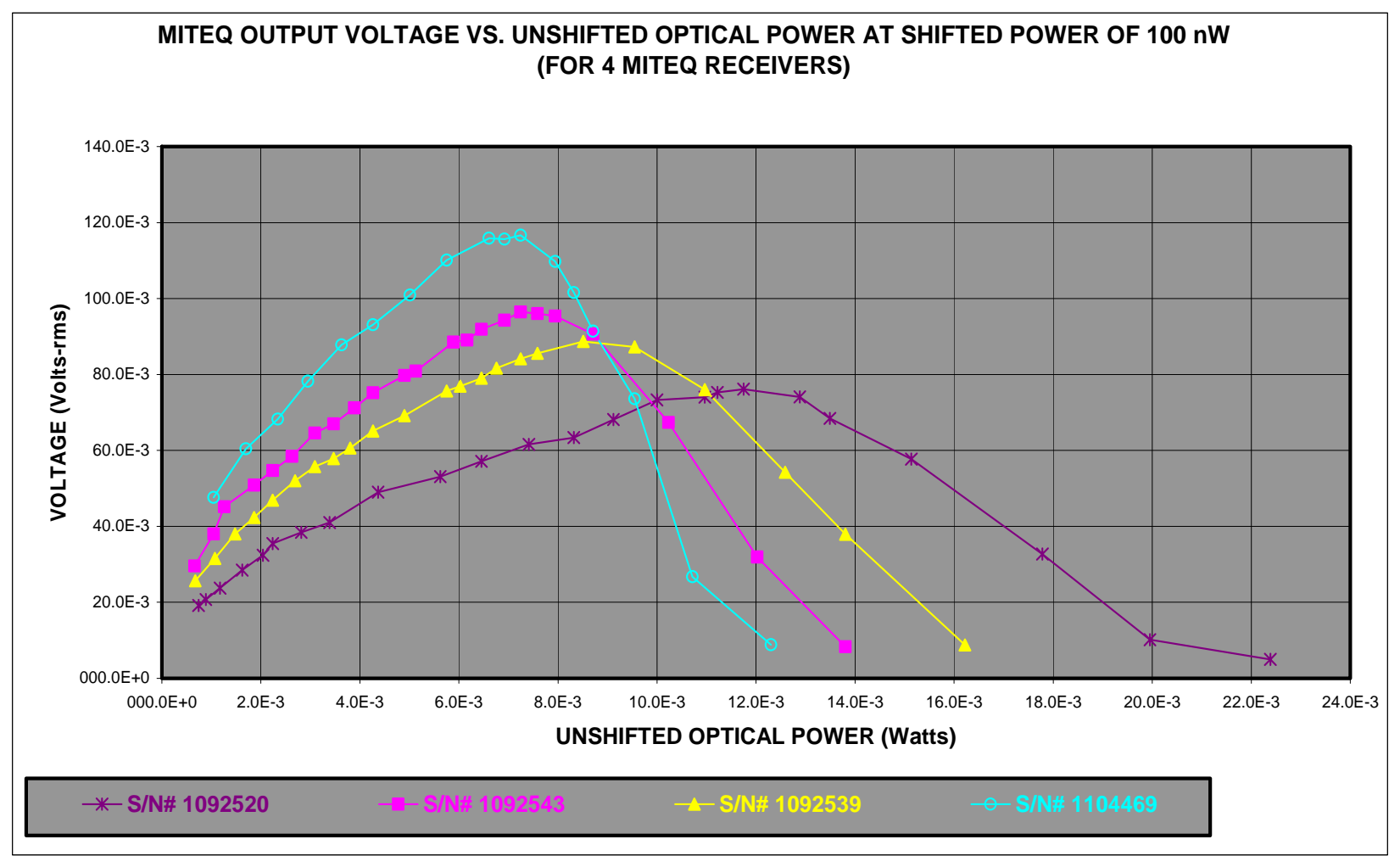

Figure 7. Transfer function for 4 different MITEQ detectors

\section{Harmonic Levels}

The second harmonic of the beat frequency was plotted for two different shifted-light levels. Figure 8 shows the fundamental frequency and the second harmonic levels. It also presents the difference (delta) in decibels between the fundamental and the second harmonic at a fixed shifted-light level of $10 \mu \mathrm{W}$. Figure 9 illustrates the second harmonic level at a 1- $\mu \mathrm{W}$ fixed shifted-light level. As the fundamental signal approaches the compression region, the relative harmonic amplitude will start to increase faster than the output. In Figure 8, the signal starts entering the compression region at $5 \mathrm{~mW}$ of input power, with a shifted-light level of $10 \mu \mathrm{W}$. In Figure 9, the signal begins entering the compression region at $9 \mathrm{~mW}$. Depending on application, a maximum desirable second harmonic level is between 35 and $40 \mathrm{dBc}$. Figures 9 and 10 demonstrate that, for the same unshifted optical power, the harmonic distortion level is proportional to the shifted optical power. In addition, the second harmonic appears highest at the compression point. 


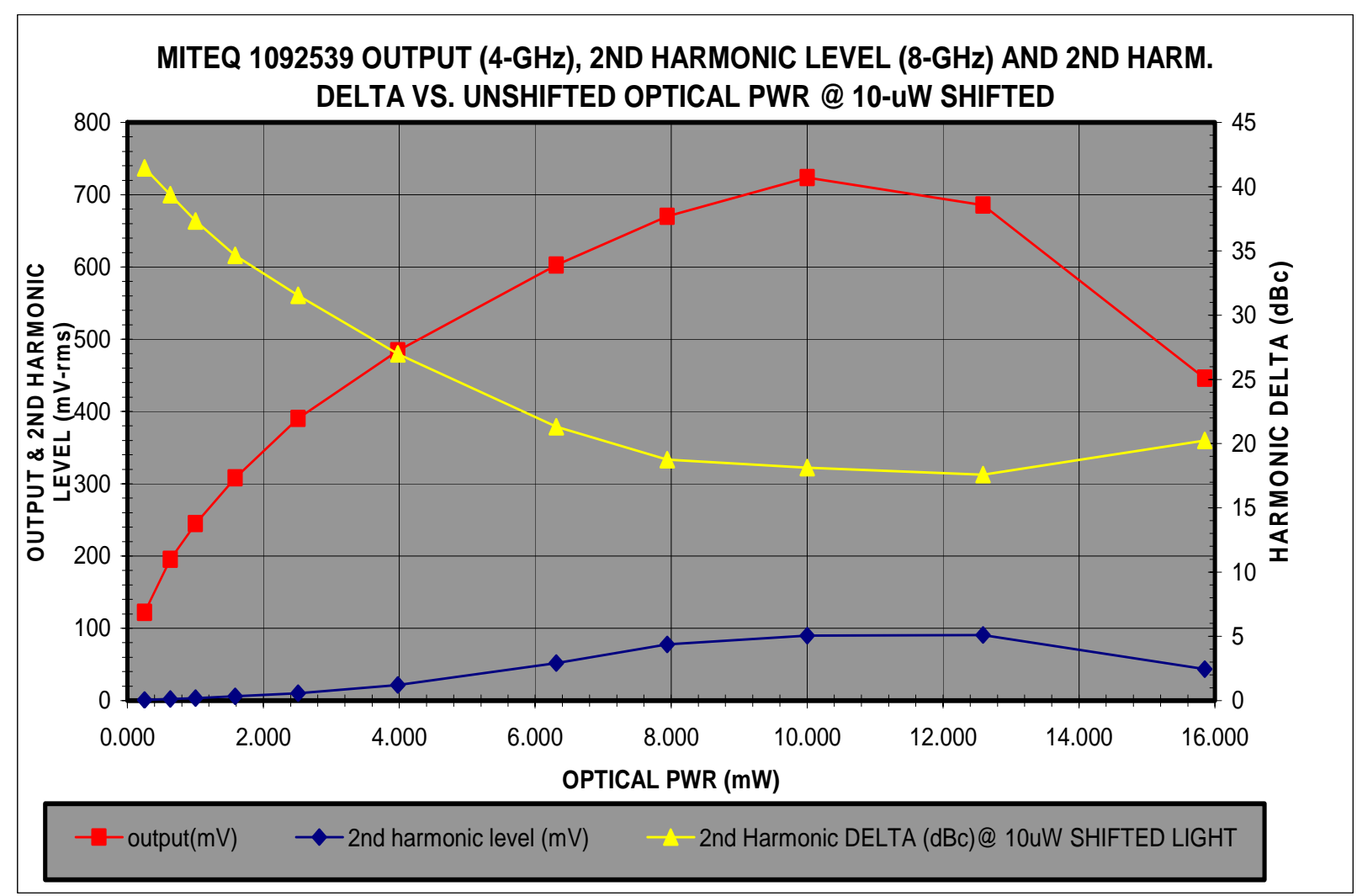

Figure 8. MITEQ harmonic distortion at 10- $\mu \mathrm{W}$ shifted power

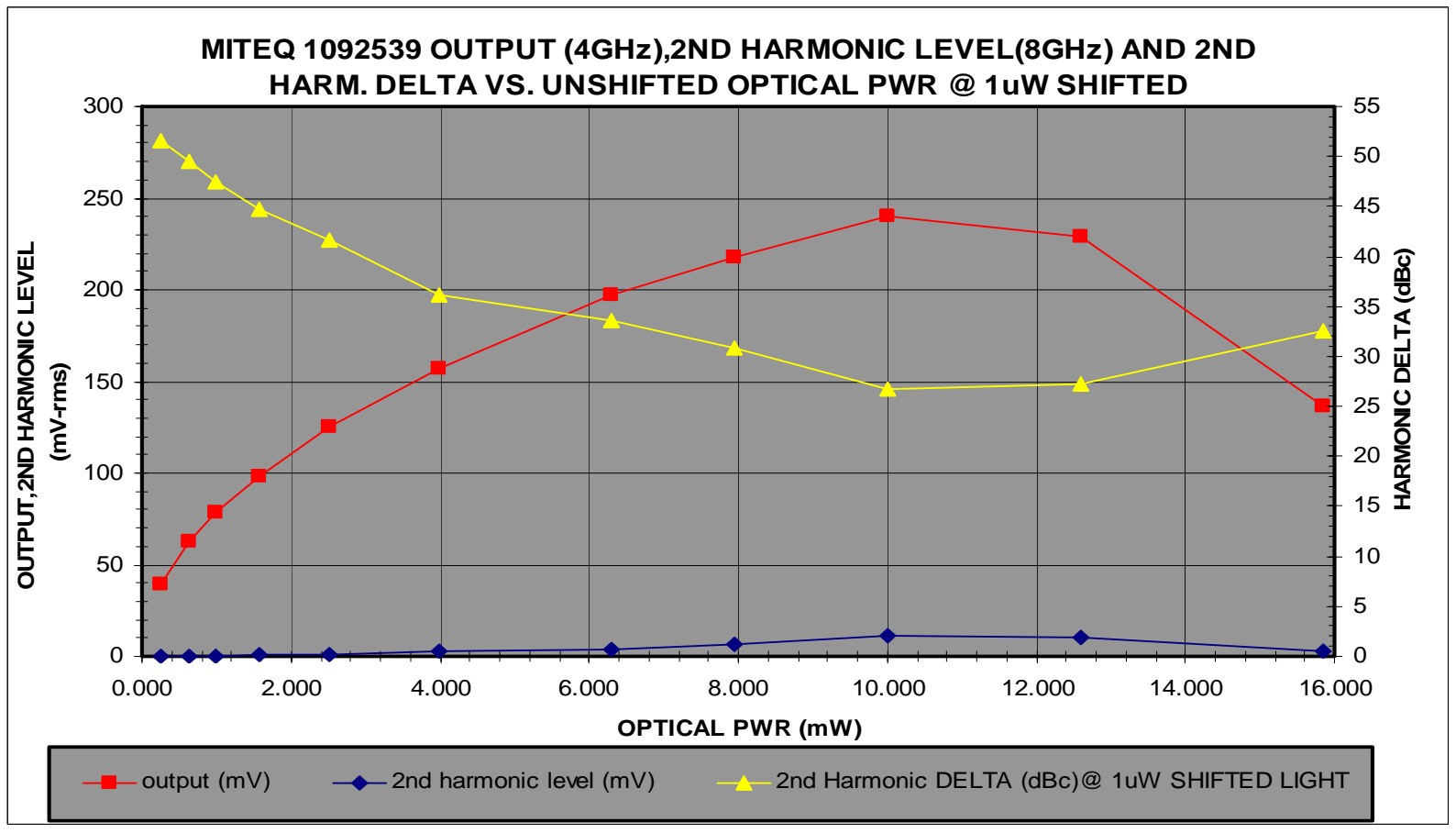

Figure 9. MITEQ harmonic distortion at $1-\mu \mathrm{W}$ shifted power 


\section{Signal-to-Noise Evaluation}

The SNR was first normalized over a 1-Hz bandwidth. The same four fixed, shifted-light levels were used (see results in Figure 11). The data show the SNR peaking at the unshifted-light level of $0 \mathrm{dBm}$. Figures 4 and 10 indicate that the optical input power for highest output voltage is not the same as the optical power level for optimum SNR. Therefore, a trade-off exists between output level and SNR. Figures 11-14 show the same SNR plots as Figure 10, except that the bandwidth was changed from $1 \mathrm{~Hz}$ to $1 \mathrm{GHz}, 4 \mathrm{GHz}, 8 \mathrm{GHz}$, and $12 \mathrm{GHz}$, respectively. Bandwidth plays an important role in optimizing the SNR for low shifted-light signals. Note that the peak SNR of the 100 -nW curve is $12 \mathrm{~dB}$ in a 4-GHz bandwidth (Figure 12). When the bandwidth doubles to $8 \mathrm{GHz}$, the SNR for the same 100-nW curve reduces to $9 \mathrm{~dB}$ (Figure 13). Generally, the SNR will increase $3 \mathrm{~dB}$ for a $50 \%$ reduction in operating bandwidth. Figure 11 shows that in order to optimize the SNR for this particular MITEQ unit, the unshifted optical power level should fall within the range of 0 to $-2.5 \mathrm{dBm}$.

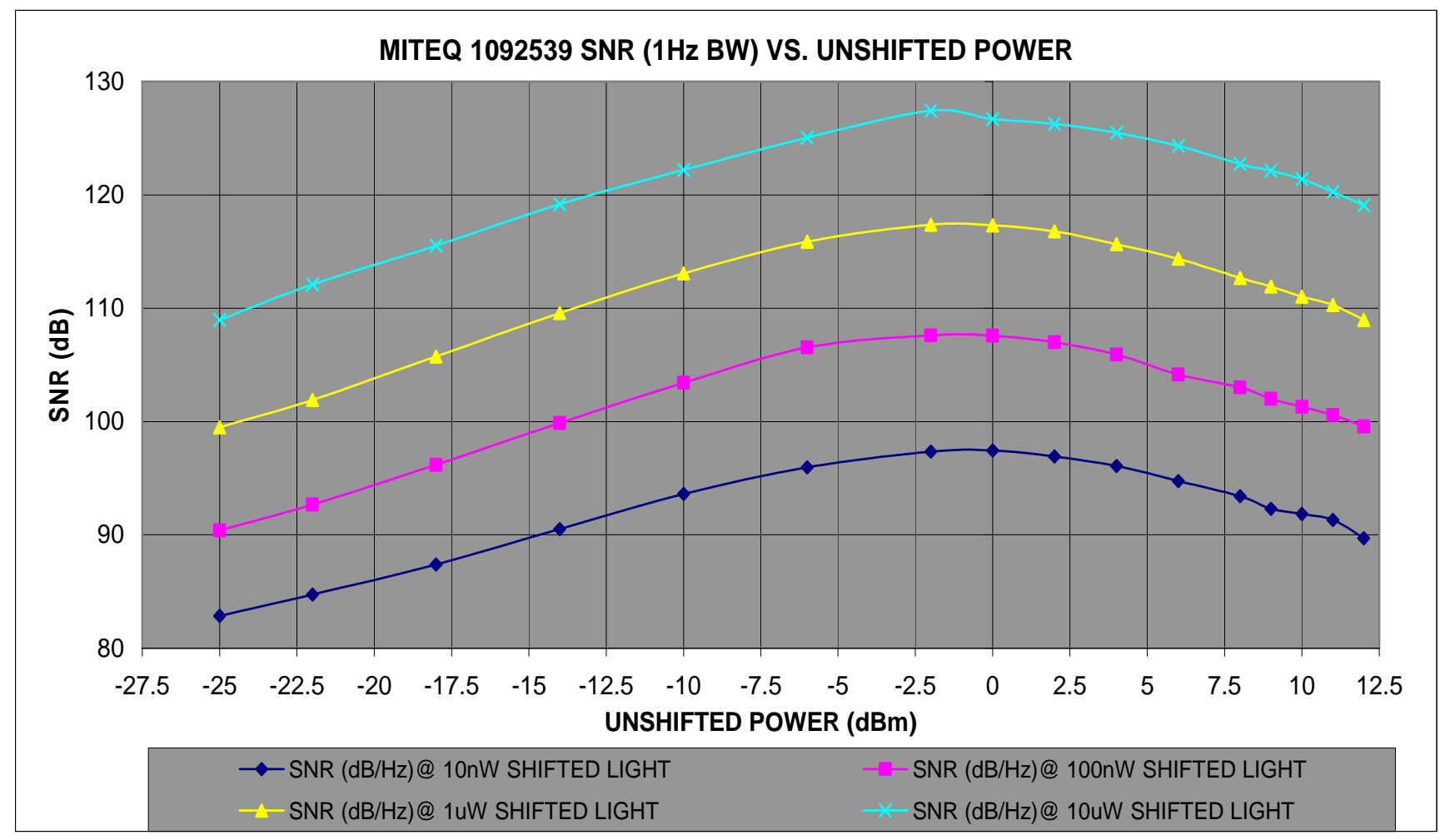

Figure 10. MITEQ signal-to-noise ratio in 1-Hz bandwidth 


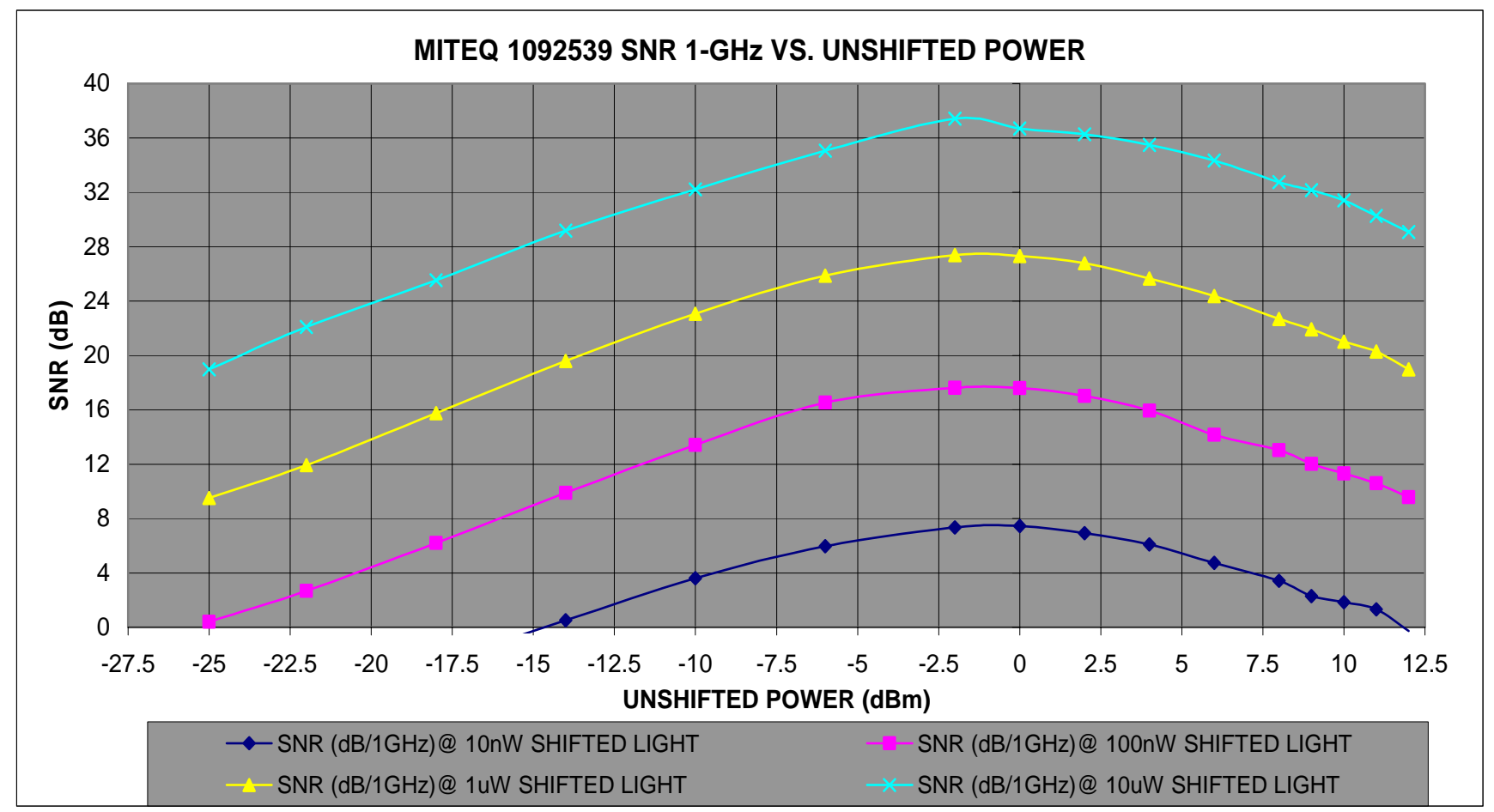

Figure 11. MITEQ signal-to-noise ratio in 1-GHz bandwidth

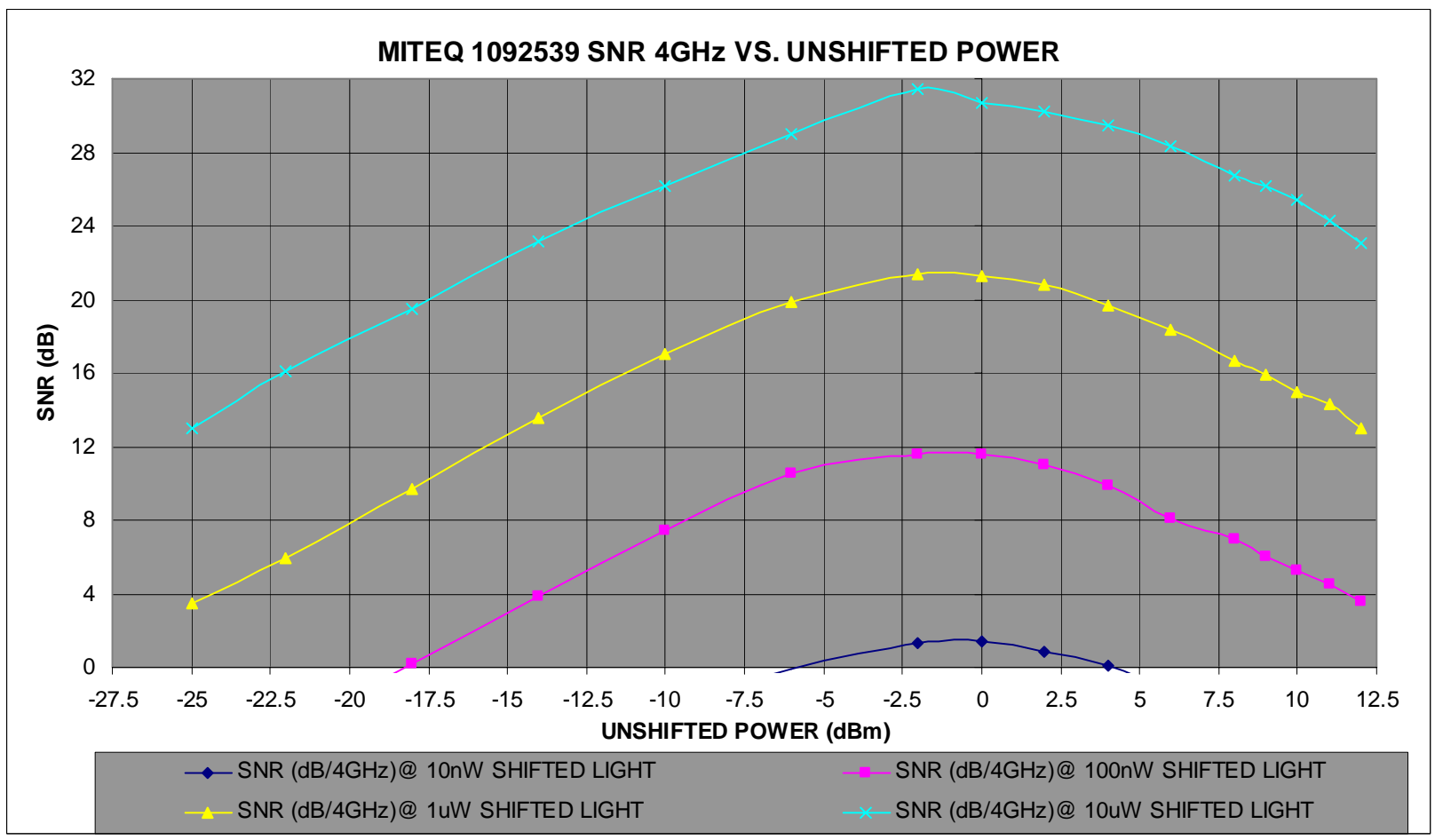

Figure 12. MITEQ signal-to-noise ratio in 4-GHz bandwidth 


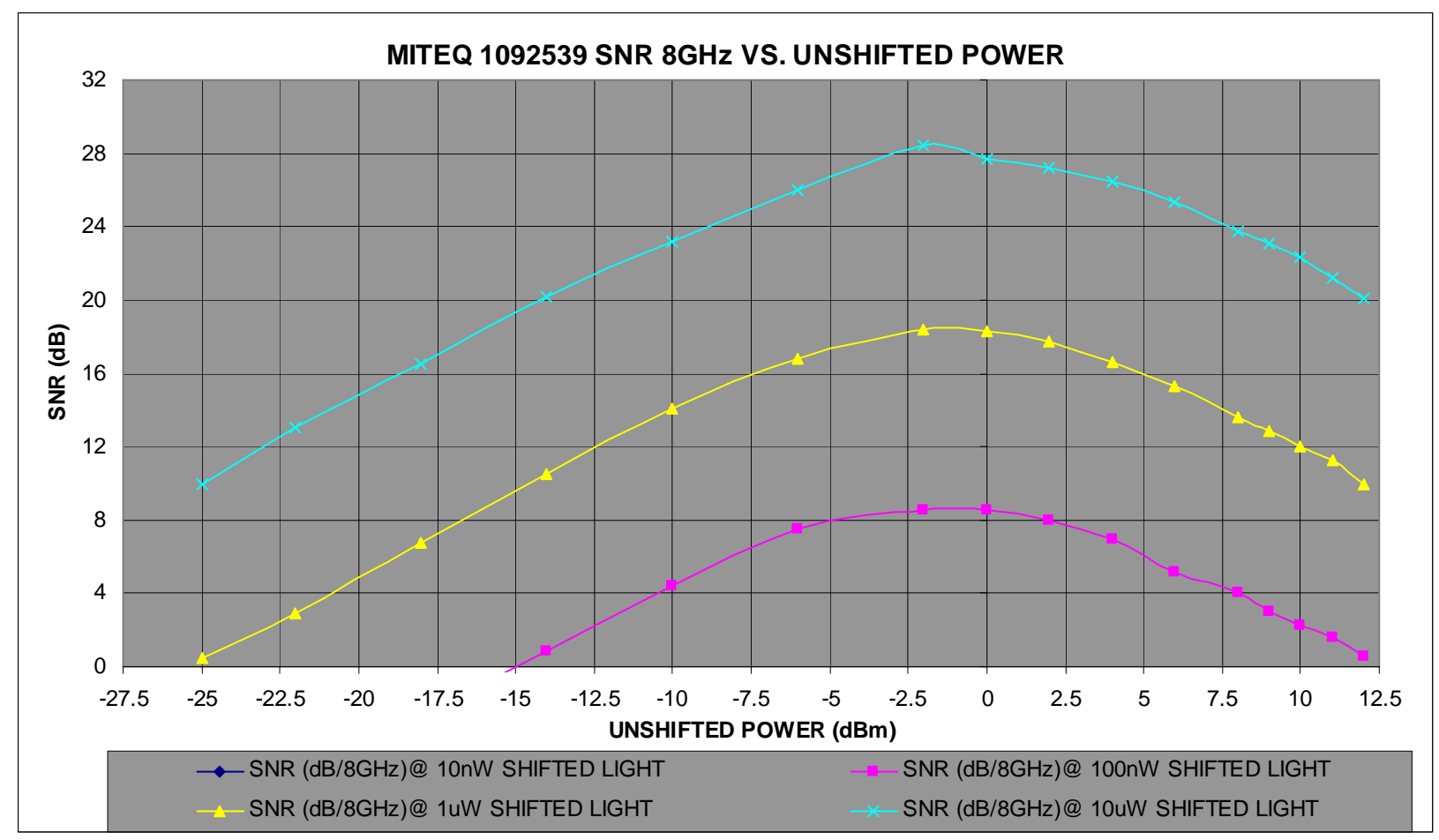

Figure 13. MITEQ signal-to-noise ratio in 8-GHz bandwidth

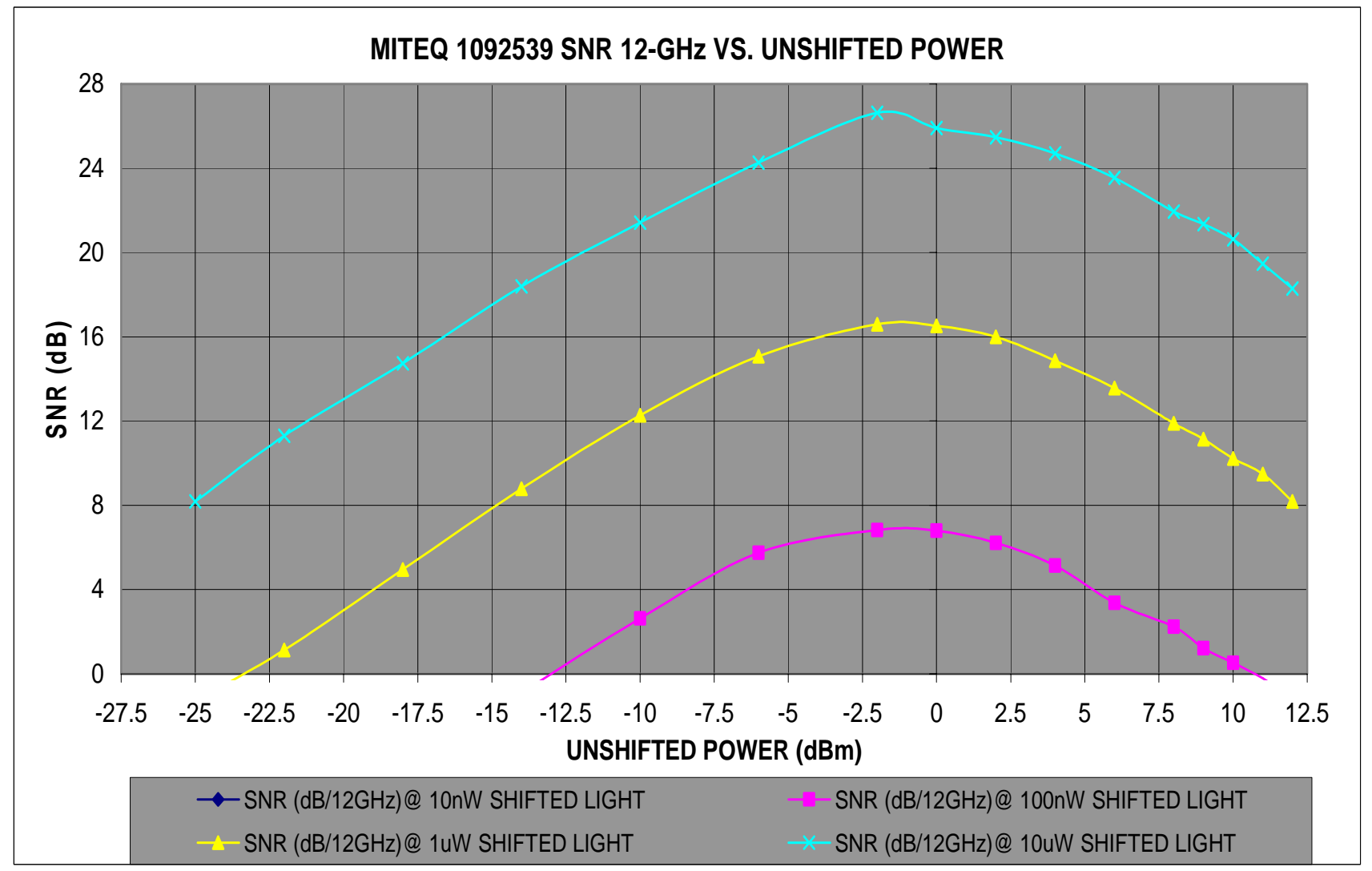

Figure 14. MITEQ signal-to-noise ratio in 12-GHz bandwidth 


\section{Conclusion}

Four MITEQ DR-125G-A detectors were lab-tested and evaluated for transfer function, second harmonic level, and SNR. The transfer function curves show that an optimum input optical power level will yield a maximum output voltage. If this optimum optical input power is exceeded, the detector will go into compression (output signal will decrease for increasing optical input power). This will also produce high second harmonic distortions (10-20 dB down from the beat frequency) and reduce the SNR, consequently decreasing the dynamic range. The optimum optical power level for output voltage differs from that of an optimum SNR, thus necessitating a trade-off between output and SNR. The optimum optical input level for output voltage varies for each MITEQ detector; it may also be temperature-dependent. Thus, in certain applications, a detector screening may need to be performed. If the detector's operating point remains within the linear portion of the curve (input optical power at $1 \mathrm{~mW}$ ), the SNR and second harmonic distortion will be optimal. An external postamplifier can be used to achieve an output signal high enough for the digitizers.

Operating the detectors at the full 12.5-GHz bandwidth may work acceptably for a shifted-light level $>1 \mu \mathrm{W}$. Sensitivity and dynamic range are usually a concern, particularly for shifted-light levels below $1 \mu \mathrm{W}$; in such cases the operational bandwidth may need to be reduced with a filter. By halving the bandwidth, the SNR will increase by $3 \mathrm{~dB}$. This, along with proper biasing, will greatly improve detector sensitivity and dynamic range.

Copyright. This manuscript has been authored by Bechtel Nevada and National Security Technologies under Contract Nos. DE-AC08-96NV11718 and DE-AC52-06NA25946 with the U.S. Department of Energy. The United States Government retains and the publisher, by accepting the article for publication, acknowledges that the United States Government retains a nonexclusive, paid-up, irrevocable, world-wide license to publish or reproduce the published form of this manuscript, or allow others to do so, for United States Government purposes.

Disclaimer. This paper was prepared as an account of work sponsored by an agency of the U.S. Government. Neither the U.S. Government nor any agency thereof, nor any of their employees, nor any of their contractors, subcontractors or their employees, makes any warranty or representation, express or implied, or assumes any legal liability or responsibility for the accuracy, completeness, or usefulness of any information, apparatus, product, or process disclosed, or represents that its use would not infringe privately own rights. Reference herein to any specific commercial product, process, or service by trade name, trademark, manufacturer, or otherwise, does not necessarily constitute or imply its endorsement, recommendation, or favoring by the U.S. Government or any agency thereof. The views and opinions of authors expressed herein do not necessarily state or reflect those of the U.S. Government or any agency thereof. 